\title{
Magnetic Susceptibility of Metals and Alloys in the Molten State*
}

\author{
By Sakae Takeuchi** and Hirohisa Endo**
}

\begin{abstract}
In the author's previous report, it was presumed that in the electronic structure of metals in the molten state that valence electrons of atoms of metals behave as free electron gas. To examine such an assumption the magnetic susceptibilities of molten metals and molten alloys were measured in wide ranges of temperatures and results were compared with theoreti cal values computed on the basis of the free electron gas model. An extremely sensitive apparatus was devised for measuring very weak magnetic susceptibilies of molten metals. Pure metals used in this experiment were $\mathrm{Ag}, \mathrm{Cd}, \mathrm{In}, \mathrm{Sn}, \mathrm{Bi}$ and $\mathrm{Sb}$ of $99.99 \%$ purity or higher, and the alloys used were $\mathrm{Bi}-\mathrm{Ag}, \mathrm{Bi}-\mathrm{Cd}, \mathrm{Bi}-\mathrm{In}, \mathrm{Bi}-\mathrm{Sn}, \mathrm{Sn}-\mathrm{Pb}$ and $\mathrm{Pb}-\mathrm{Sb}$.

It was shown that observed magnetic susceptibilities were always independent of temperature in pure metals and also in alloys, and were in good agreement with the calculated values given by the sum of the paramagnetic susceptibility contributed by the free electron gas and the diamagnetic susceptibility of the ion cores.
\end{abstract}

(Received February 13, 1962)

\section{Introduction}

As reported previously(1)(2), when a metal is melted, the crystal structure is broken down, and it may be assumed that the Brillouin zone structure associated with the electronic energy is destroyed thereby, permitting

** The Research Institute for Iron, Steel and Other Metals, Tohoku University, Katahiro-cho, Sendai, Japan.

* This Paper was read at the Spring Meeting of The Japan Institute of Metals, 3 April; 1961. Tokyo, Japan.

(1) S. Takeuchi and. H. Endo: Trans. JIM. 2 (1961), 188.

(2) S. Takeuchi and H. Endo: Trans. JIM. 2 (1961), 189. the valence electrons to behave as free electrons among the randomly arranged ions in thermal motion. In the author's previous report ${ }^{(2)}$, with the aim of verifying this assumption, the Hall coefficient was measured in several metals in the molten state, and it was found that the values obtained were always negative, and were nearly independent of temperature and agreed well with the theoretical values computed by the free electron model.

Now, if the valence electrons in molten metals do behave as free electron gas, the magnetic susceptibility 
of such electron gas should naturally take the value computed by Pauli(3) and Landau's formula(4), that is, the magnetic susceptibility of the free electron gas will be given by subtracting the magnetic susceptibility of the ions from the measured susceptibility of the molten metals and consequently should have a value independent of temperature. For the above reasons, in this study the magnetic susceptibility of several metals whose valencies ranged from monovalent to quinquevalent was measured and the values obtained were compared critically with the theoretical values calculated by the free electron gas model.

\section{Magnetic Susceptibilities of Pure Metals in the free Electron Gas Model}

The magnetic susceptibility of a molten metal can be expected to be given as the sum of the magnetic susceptibility of the free electron gas consisting of the valence electron and that of the ion cores as stated above. Free electron gas can contribute to the magnetic susceptibility in two ways. The first one arises from the diamagnetism due to the translational motion of the degenerated free electron gas, which is given by Landau's formula

$$
M_{D}=\frac{n_{0} \mu^{2}}{2 \varepsilon_{0}}\left\{1+\frac{\pi^{2}}{12}\left(\frac{k T}{\varepsilon_{0}}\right)^{2}\right\},
$$

where $\mu$ denotes the Bohr magneton, $\varepsilon_{0}$ Fermi energy and $n_{0}$ the number of free electrons per atom. In equation (1) $\varepsilon_{0} \gg k T$, the second term can be neglected. If, for the sake of convenience in comparison with experimental results, the magnetic susceptibility per unit mass $\chi$ is introduced, the above formula can be rewritten as

$$
\chi_{L}=0.623 n_{0}^{1 / 3} \rho^{-2 / 3} W^{-1 / 3} \times 10^{-6} \text { C.G.S. unit, }
$$

where $\rho$ is the density of the molten metal and $W$ its atomic weight. The second arises from the paramagnetism due to the electron spin. This paramagnetic susceptibility is just three times as large as Landau's diamagnetic susceptibility. Therefore, the share in the total magnetic susceptibility contributed by the free electron gas is $2\left|\chi_{L}\right|$, and consequently, the total magnetic susceptibility of the molten metal is given by

$$
\chi=\chi_{\text {electron }}+\chi_{\text {ion }}=2\left|\chi_{L}\right|+\chi_{\text {ion }} \text {. }
$$

$\chi_{\text {ion }}$ may be determined by Langevin's formula ${ }^{(5)}$, but can also be obtained from the magnetic susceptibility of the salts of the metal in question.

\section{Apparatus for Measuring Magnetic Susceptibility}

The magnetic susceptibility of molten metals is generally very weakly diamagnetic, of the order of $10^{-7}$ to $10^{-8}$. When the high temperature required is considered in addttion with the above reason, it makes its measurement very difficult. In this study, the present authors adopted a balance method in which the change in magnetic force acting upon the specimen suspended in a nonuniform magnetic field generated by a Weiss electromagnet was measured by means of a highly sen-

(3) Pauli: Zeit. Phys. 41, (1927), 81.

(4) Landau: Zeit. Phys., 64 (1930), 629.

(5) P. Langevin : Ann. Chem. Phys., 5 (1905), 70. sitive balance. For further increasing the sensitivity of the balance, a piece of tin foil was attached to the tip of the needle indicator showing the inclination of the balance, another piece of tin foil fixed parallel to the former, the capacity between the two parallel pieces of tin foil was changed by the movement of the indicator, which was caused by the movement of the balance arm and this change in capacity was detected and recorded by a miero-capacity detection circuit and recorder, from which the weak diamagnetism of the molten specimen was measured. Fig. 1 shows the apparatus. Two or three grams of the molten metal was sealed in a thin

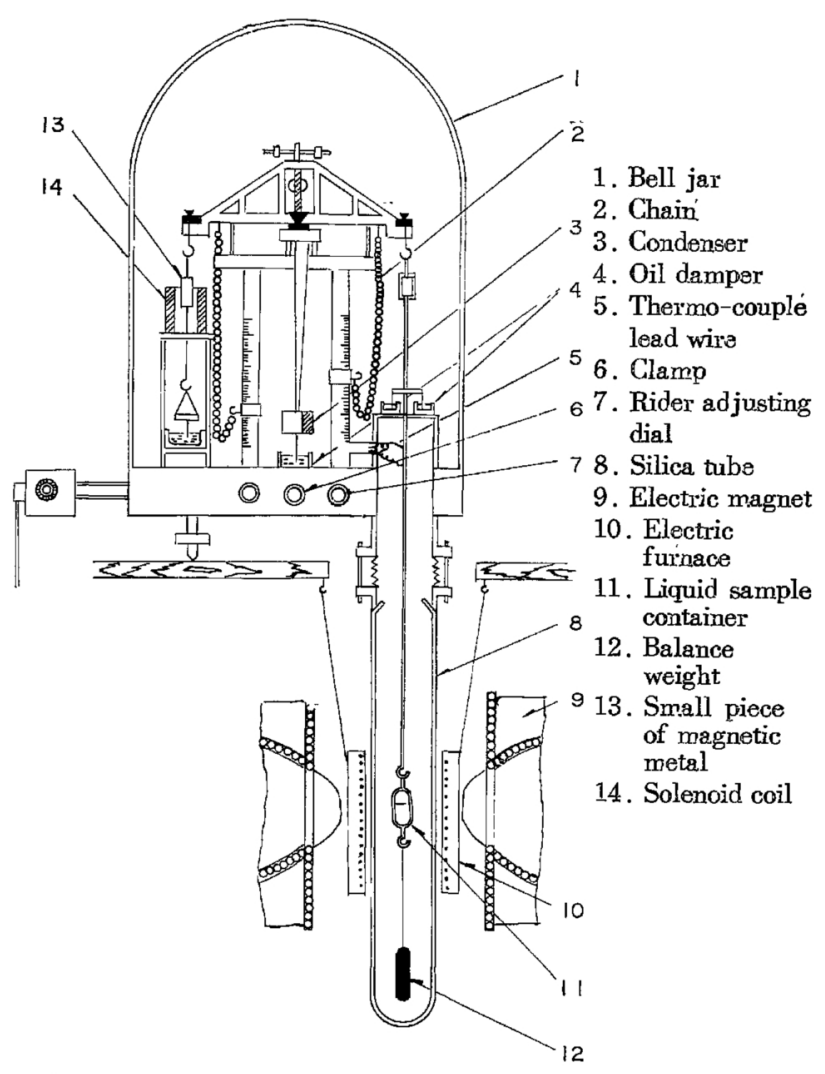

Fig. 1 Exiremely sensitive measuring apparatus for measuring the magnetic susceptibility of molien metals.

transparent quartz tube of $10 \mathrm{~mm}$ inner diameter and $10 \mathrm{~mm}$ length for use as a specimen. The balance system and the specimen were placed in a vacuum container, and the balance clamp and adjustment of the zero point of the needle was operated from outside the vacuum system.

The sensitivity of the balance was of the order of $0.7 \times 10^{3}$ degree per $\mathrm{gram}$ in the inclination of the balance arm. The specimen was suspended near the place in the magnetic field where the maximum value of $H \frac{\partial H}{\partial Z}=3 \times 10^{6} \frac{\mathrm{Oe}^{2}}{\mathrm{~cm}}$ could be obtained. The magnetic susceptibility $-0.702 \times 10^{-6}$ of Benzene purified by repeated distillation was used as a standard. The accuracy of the measurement could be kept within $\pm 3 \%$.

\section{Experimental Results}

The specimens measured were silver, cadmium, indium, tin, lead, antimony and bismuth chosen because of their low melting points. The purity of all of the metals was $99.99 \%$ or higher. 
The magnetic susceptibility temperature curve of $\mathrm{Ag}$ is shown in Fig. 2, $\chi=-0.26 \times 10^{-6}$ remained nearly constant independent of temperature from room temperature to just below the melting point, but abruptly

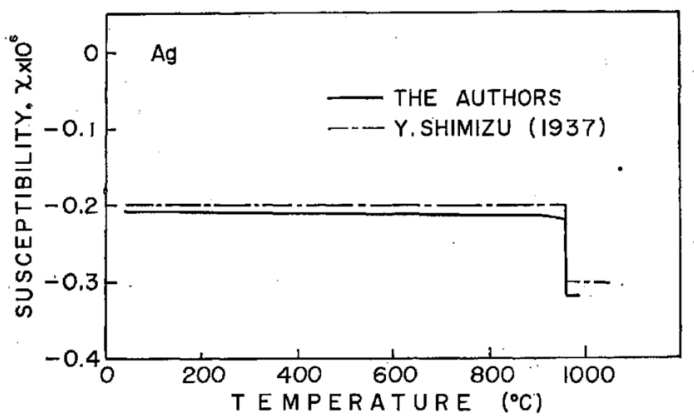

Fig.2 Temperature dependence of magnetic susceptibility of $\mathrm{Ag}$ in the solid and the molten state.

changed to $\chi=-0.32 \times 10^{-6}$ upon melting and thereafter remained constant in spite of further increases of $t^{\text {emperature. The measured values obtained by Shimizu }}{ }^{(6)}$ are also indicated for comparision in Fig. 2. The same curve of the divalent metal Cd is shown in Fig. 3, Endo(7)

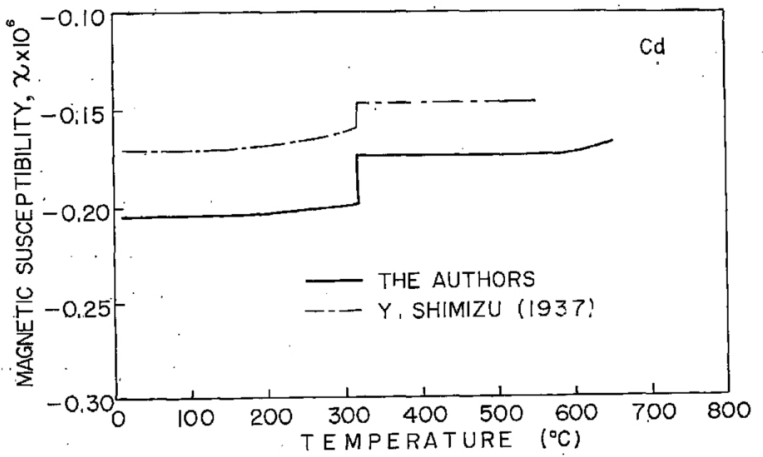

Fig.3 Temperature dependence of magnetic susceptibility of $\mathrm{Cd}$ in the solid and the molten state.

has reported a similiar measurement. Solid Cd shows diamagnetism of $\chi=-0.197 \times 10^{-6}$ near the melting point and of $\chi=-0.177 \times 10^{-6}$ after melting, which remained constant thereafter till the boiling point $\left(765^{\circ} \mathrm{C}\right)$ was approached, when the value of diamagnetism began to drop slightly, indicating a paramagnetic tendency. Indium, as seen in Fig. 4 showed diamagnetism of the susceptibility $\chi=-0.07 \times 10^{-6}$ at room temperature but it changed to a constant value of $-0.056 \times 10^{-6}$ after melting. As shown in the same figure, Sn was paramagnetic at room temperature, but turned diamagnetic upon melting and the value remained constant in the molten state regardless of temperature change. The temperature dependence of the magnetic susceptibility for $\mathrm{Pb}$ is also shown in Fig. 4, where $\chi$ rose almost linearly with increase of temperature in the solid state, but upon melting, it became $-0.072 \times 10^{-6}$ and remained independent of temperature. $\mathrm{Bi}$, as shown in Fig. 5, in the solid state, showed a very large diamagnetism of $\chi=-1.02 \times 10^{-6}$ at room temperature which decreased rapidly with increasing temperature and with an abnormally steep break at the melting point abruptly to a very small value $\chi=-0.052 \times 10^{-6}$. In $\mathrm{Sb}$, the diamagnetism in the solid state changed with increase

(6) Y. Shimizu : Sci. Rep. Tohoku, 25 (1936), 921.

(7) H. Endo: Sci. Rep. Tohoku, 16 (1927), 201. of temperature; but in the molten state the value of $\chi$ remained at a low level, $-0.02 \times 10^{-6}$, as shown in Fig. 5 , where the values obtained by $\mathrm{Bush}^{(8)}$ and Endo ${ }^{(7)}$ are indicated.

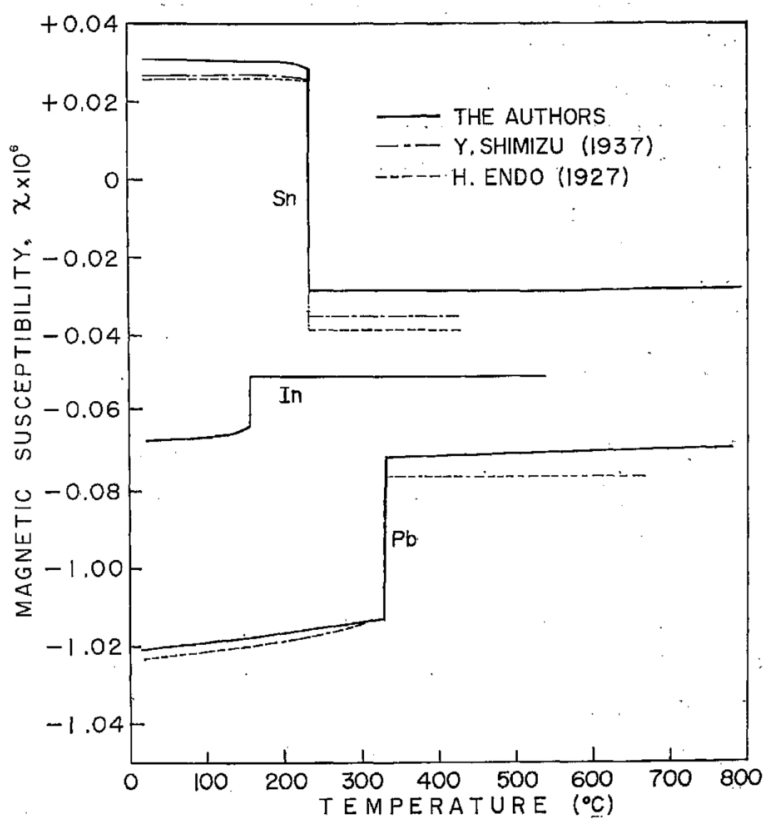

Fig.4 Temperature dependences of magnetic susceptibilities of $\mathrm{In} . \mathrm{Sn}$ and $\mathrm{Pb}$ in the solid and the molten state.

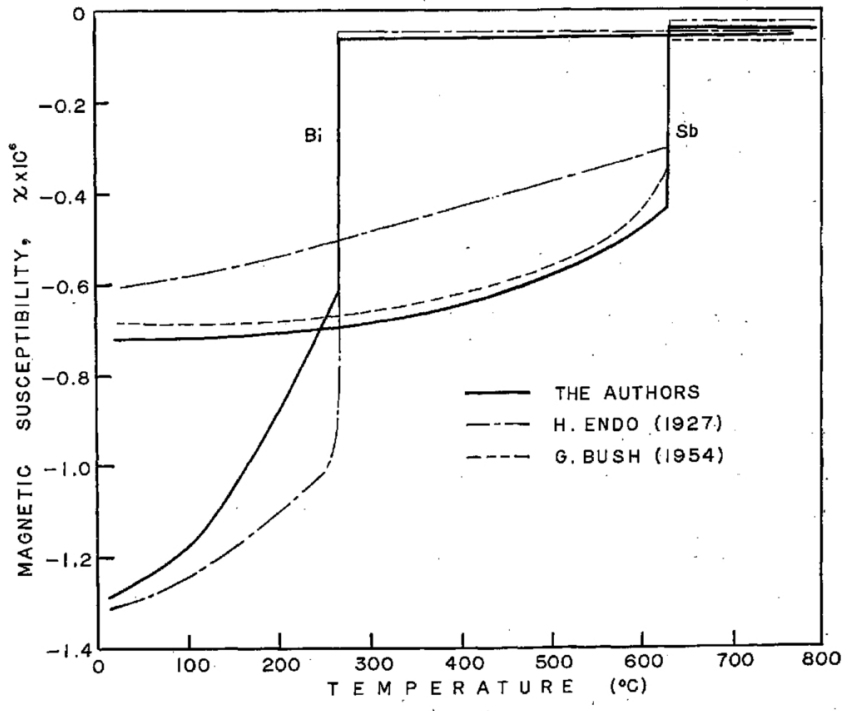

Fig.5 Temperature dependences of magnetic susceptibilities of $\mathrm{Bi}$ and $\mathrm{Sb}$ in the solid and the molten state.

\section{Discussion of the Experimental Results}

Since the magnetic susceptibilities obtained in the above measurements represent $\chi_{\text {electron }}+\chi_{\text {ion, }}$ the latter value must be subtracted from the measured values to obtain those due to the free electron gas. It is, however, not so easy to determine the correct value of $\chi_{\text {ion }}$. Calculations of the diamagnetism for ion cores have been reported by Hartree ${ }^{(9)}$, Stoner ${ }^{(10)}$, Slater ${ }^{(11)}$ Brindley ${ }^{(12)}$ and Angus(13), but values calculated by them do not agree very well, also the values deduced
(8) G. Bush and O. Vogt: Helv. Phys. Acta 27 (1954), 241.
(9) D. R. Hartree: Proc: Camb. Phil. Soc., 24 (1928), 89, 111.
(10) E. C. Stomer : Proc. Leeds Phil. Soc., 1 (1929), 484.
(11). J. C. Slater: Phys. Rev., 36 (1930), 57 .
(12) G.W. Brindley : Phil. Mag., 11 (1931), 786.
(13) W. R. Angus : Proc. Roy. Soc. Lond., A136 (1932); 569. 
from the measured magnetic susceptibilities of various salts are not very accurate. In this study, the required values of metallic ions are cited from the tables of Mott $^{(12)}$ and Van Vleck(15). In Table 1 are shown the results for the measured values of magnetic susceptibilities of metals in the solid state $\left(20^{\circ} \mathrm{C}\right)$ and in the molten state, thte values of $\chi_{\text {ion }}$ and the theoretical values representing the sum of $\chi_{\text {ion }}$ and $2\left|\chi_{L}\right|$ calculated by Landau's formula (2) on the assumption of free electron gas. As seen in Table 1 , the theoretical values always agree well with measured values in the molten state of any metals, but not in the solid state. contains the terms $\left\{\frac{n_{\mathrm{A}}(1-x)+n_{\mathrm{B}} x}{W_{\mathrm{A}}(1-x)+W_{\mathrm{B}} x}\right\}^{1 / 3}$ and $\left(\rho_{\mathrm{AB}}^{0}\right)^{-2 / 3} \times$ $\left(1-\frac{2}{3} \frac{\Delta \rho}{\rho_{\mathrm{AB}}^{0}}\right)$ in place of $\left(\begin{array}{c}n_{0} \\ W^{-}\end{array}\right)^{1 / 3}$ and $\rho^{-2 / 3}$ respectively. The density $\rho$ is not generally represented by the arithmetic average $\rho_{\mathrm{AB}}^{0}(x)$ of the densities of $\mathrm{A}$ and $\mathrm{B}$, but shows a slight deviation $\Delta \rho(x)$ from $\rho_{\mathrm{AB}}^{0}$;

$$
\rho^{-2 / 3}=\left\{\rho_{\mathrm{AB}}^{0}(x)+\Delta \rho(x)\right\}^{-3 / 2} \fallingdotseq\left(\rho_{\mathrm{AB}}^{0}\right)^{-2 / 3}\left(1-\frac{2}{3} \frac{\Delta \rho}{\rho_{\mathrm{AB}}^{0}}\right)
$$

For example, from the observed density-concentration curve $^{(16)} \rho_{\mathrm{AB}}^{0}$ and $\Delta \rho$ at $20 \% \mathrm{Bi}-\mathrm{Cd}$ alloy are obtained as follows

Table 1 Magnetic susceptibilities of several kinds of metals in the molten state, measured and calculated from Landau's formula.

\begin{tabular}{|c|c|c|c|c|c|c|c|c|}
\hline \multirow{2}{*}{ Metal } & \multicolumn{6}{|c|}{ Calculated $\chi \times 10^{6}$} & \multicolumn{2}{|c|}{ Observed $\chi \times 10^{6}$} \\
\hline & $\rho$ & $W$ & $n_{0}$ & $2\left|\chi_{L}\right| \times 10^{6}$ & $\chi_{i} \times 10^{6 *}$ & $2\left|\chi_{L}\right|+\chi_{i}$ & $\chi$ (liquid) & $\chi\left(\right.$ solid $\left.20^{\circ} \mathrm{C}\right)$ \\
\hline $\begin{array}{l}\mathrm{Ag} \\
\mathrm{Cd} \\
\mathrm{In} \\
\mathrm{Sn} \\
\mathbf{P b} \\
\mathrm{Bi} \\
\mathrm{Sb}\end{array}$ & $\begin{array}{c}9.301 \\
8.020 \\
7.035 \\
7.00 \\
10.67 \\
10.07 \\
6.504\end{array}$ & $\begin{array}{l}107.88 \\
112.41 \\
114.82 \\
118.70 \\
207.21 \\
209.00 \\
121.76\end{array}$ & $\begin{array}{l}1 \\
2 \\
3 \\
4 \\
4 \\
5 \\
5\end{array}$ & $\begin{array}{l}0.0594 \\
0.0802 \\
0.1008 \\
0.1094 \\
0.0690 \\
0.0766 \\
0.1236\end{array}$ & $\begin{array}{l}-0.399 \\
-0.200 \\
-0.161 \\
-0.199 \\
-0.123 \\
-0.137 \\
-0.164\end{array}$ & $\begin{array}{l}-0.34 \\
-0.12 \\
-0.06 \\
-0.09 \\
-0.06 \\
-0.06 \\
-0.04\end{array}$ & $\begin{array}{l}-0.32 \\
-0.18 \\
-0.06 \\
-0.03 \\
-0.07 \\
-0.05 \\
-0.02\end{array}$ & $\begin{array}{l}-0.26 \\
-0.21 \\
-0.07 \\
+0.03 \\
-0.12 \\
-1.02 \\
-0.55\end{array}$ \\
\hline
\end{tabular}

The temperature dependence of the magnetic susceptibility follows $\left(k T / \varepsilon_{0}\right)^{2}$ by the formula (1), but since within the range of temperature in this experiment $k T \ll \varepsilon_{0}$, the term $\left(k T / \varepsilon_{0}\right)^{2}$, can be completely neglected. Thus, the temperature dependence may result trom the temperature change in the density of the molten metal, but since the temperature coefficient $\frac{1}{\rho_{0}} \frac{d \rho}{d T}$ of the density is only of the order of $10^{-4}$ in molten metals, any change in density within the temperature ranges of the measurement would fall within the range of errors of measurement and therefore would remain undetected unless the temperature rises near the critical point or boiling point. As shown in Fig. 3, the value in Cd begins to show a temperature dependence near $650^{\circ} \mathrm{C}$, and this exceptional behavior seems to correspond to the anormaly observed in the density of molten Cd near $650^{\circ} \mathrm{C}$.

From these results, one can conclude that in the molten state of metals, the Brillouin Zone structure associated with electronic energy known in the solid crystal is destroyed and all of the valence electrons behave as free electron gas.

\section{Magnetic Susceptibility of Molten Alloys}

If the alloy contains no compounds, the contribution of valence electrons in molten alloys to the magnetic susceptibility would be naturally in compliance with the formula of Landau. In alloys, however, the terms $\left(n_{0} / W\right)^{1 / 3}$ and $\rho^{-2 / 3}$ in formula (2) do not show a simple linear relation to the concentration of the compont metals. Let $n_{\mathrm{A}}$ and $n_{\mathrm{B}}$ be the number of valence electrons, $W_{\mathrm{A}}$ and $W_{\mathrm{B}}$ the atomic weight, respectively, of the component metal atoms and 1-x the concentration of $\mathrm{A}$ and $x$ that of $B$ in the binary alloy A-B. Formula (2) for the alloy

(14) N.F. Mott: Theory of Properties of Metals and Alloys, (1934).

(15) J. H. Van Vleck: Theory of Electric and Magnetic Susceptibilities, Oxford, (1935).

$$
\frac{|\Delta \rho|}{\rho_{\mathrm{AB}}^{0}} \fallingdotseq 0.02,
$$

the deviation $\Delta \rho$ amounts only to a few percent at the most. Only a very low percentage error would occur if the average of the densities of the component metals is used in computing the change in magnetic susceptibility due to the change in the concentration of the alloying elements. But the factor $\left\{\frac{n_{\mathrm{A}}(1-x)+n_{\mathrm{B}} x}{W_{\mathrm{A}}(1-x)+W_{\mathrm{B}} x}\right\}^{1 / 3}$ does not change in a linear relation to concentration $x$, because of $\frac{n_{\mathrm{B}}}{n_{\mathrm{A}}} \neq \frac{W_{\mathrm{B}}}{W_{\mathrm{A}}}$. Therefore, it may be impossible to express the magnetic susceptibility of a molten alloy by the average of the respective susceptibility of the alloying element. Upon actual calculation, it was found that the magnetic susceptibility-concentration curves in the binary alloys of $\mathrm{Bi}-\mathrm{Ag}$ and $\mathrm{Bi}-\mathrm{Cd}$ systems are convex upwards and in alloys of the $\mathrm{Bi}-\mathrm{Sn}$ and $\mathrm{Sb}-\mathrm{Pb}$ systems are convex down-wards, while in alloys of metal of equal valence and not much different in atomic weight, the curves are nearly linear.

The measurements of the magnetic susceptibilities of molten alloys, $\mathrm{Bi}-\mathrm{Ag}, \mathrm{Bi}-\mathrm{Cd}, \mathrm{Bi}-\mathrm{In}, \mathrm{Bi}-\mathrm{Sn}, \mathrm{Sn}-\mathrm{Pb}$ and $\mathrm{Sb}-\mathrm{Pb}$, were performed by the same method as given in $:$ III.

In Fig. 6 are shown the observed and calculated magnetic susceptibilities of the molten alloy Bi-Ag. The distribution of the observed values marked by open circles shows a slight convexity upwards. It was also found that the observed value the magnetic susceptibility of the alloy in the molten state was affected very little by the change in temperature. The solid line in the figure represents the theoretical curve calculated by assuming that the contribution $\chi_{\text {electron }}$ of the free electron gas in the alloy is given by Landau's formula (2) for

(16) Y. Matuyama: Sci. Rep. Tohoku, 16 (1927), 343. 
alloys, the diamagnetic susceptibility of ion cores $\chi_{\text {ion }}$ is represented by subtracting the calculated $\chi_{\text {electron }}$ for respective alloying elements, $\mathrm{Bi}$ and $\mathrm{Ag}$ from the observed susceptibility $\chi$ for the pure element and $\chi_{\text {ion }}$ for the alloy can be given by the average of the values

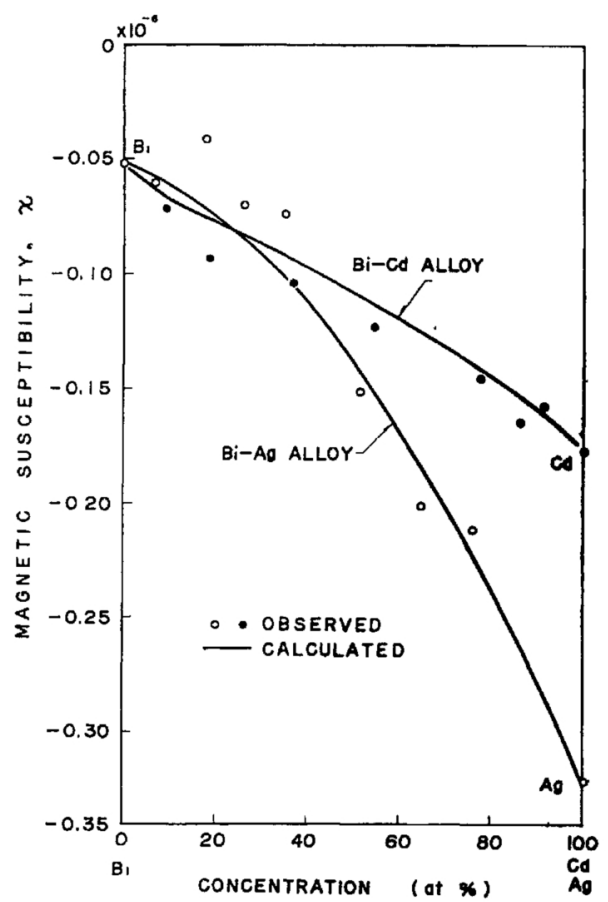

Fig.6 Plots of magnetic susceptibilities of $\mathrm{Bi}-\mathrm{Ag}$ and $\mathrm{Bi}-\mathrm{Cd}$ molten alloys in relation to concentration, measured and calculated.

of the component metals obtained as above. As shown in the figures, theoretical curves and the distribution of the observed values are found to be in very good agreement.

The same relation in the molten alloy system of $\mathrm{Bi}-\mathrm{Cd}$ is shown in Fig. 6 . The theoretical curve shown by a

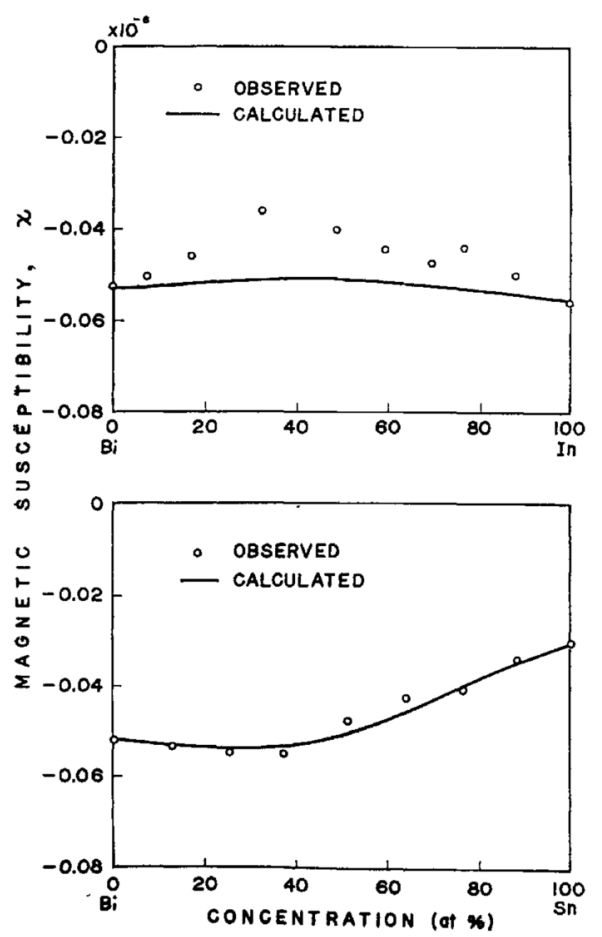

Fig.7 Plots of magnetic susceptibilities of $\mathrm{Bi}-\mathrm{In}$ and $\mathrm{Bi}$ molten alloys in relation to concentration, measured and calculated. solid line is in agreement with the observed values. In Fig. 7 the measured values and the theoretical curve for the molten alloy system of $\mathrm{Bi}$-In are also plotted. The distribution of the measured values seems to be convex upwards, and the theoretical curve is very slightly

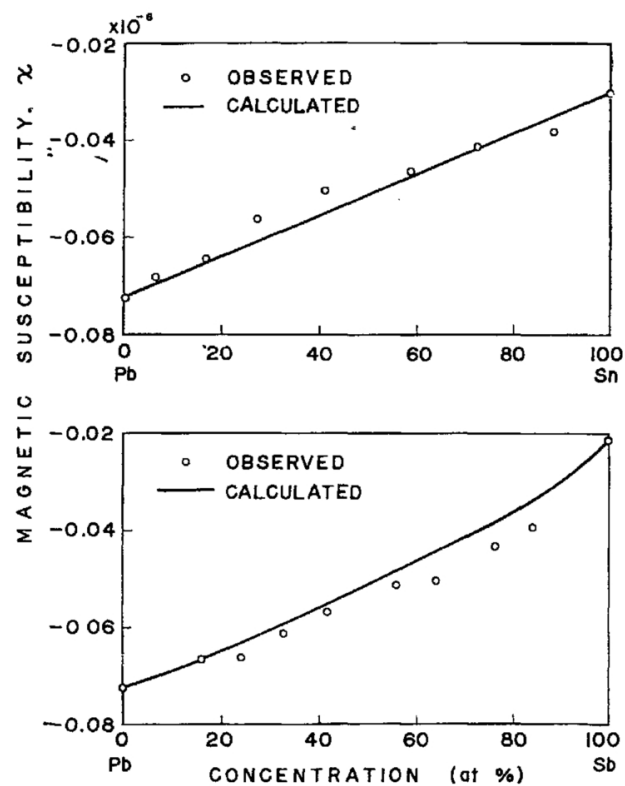

Fig.8 Plots of magnetic susceptibilities of $\mathrm{Pb}-\mathrm{Sn}$ and $\mathrm{Pb}-\mathrm{Sb}$ in relation to concentration, measured and calculated.

convex upward but is nearly straight. From the fact that the magnetic susceptibility of pure $\mathrm{Bi}$ is nearly the same as that of pure In and they are very small in magnitude, such a slight disagreement between calculation and observation may be due to the influence of the experimental errors. The observed susceptibility for each composition of alloy was independent of temperature. The measured values of the magnetic susceptibility for the respective compositions of the molten alloy of Bi-Sn agree very closely with the calculated values and lay nearly on the calculated convex curve of the susceptibility-composition, as shown in Fig. 7.

In the molten alloy $\mathrm{Sn}-\mathrm{Pb}$, the theoretical curve is nearly linear, but in this case, the measured values are scattered on both sides of a straight line within a narrow range, indicating very good agreement between the calculated and observed values as shown in Fig. 8. The relation of magnetic susceptibility versus composition in the molten alloy of $\mathrm{Pb}-\mathrm{Sb}$ also, as shown in Fig. 8, describes a concave curve theoretically and the observed value for each composition is found on the curve, indicating good agreement. As shown and described above, in molten alloys the valence electrons of component atoms form a degenerated free electron gas and the magnetic susceptibility of alloy follows the modified Landau formula.

Thus, the results of the measurements of the magnetic susceptibility in pure metals and alloys in the molten state, in combination with the observed results for the Hall coefficients of the molten metals, as repoeted previously, indicate that all of the valence electrons of all of the atoms in molten metals behave as free electrons and produce the electronic structure of a degenerated free electron gas. 


\section{Summary}

1. When a solid metal is melted, the crystal structure is broken down and it may be assumed in metals in the molten state that the collapse of the Brillouin Zone structure associated with electronic energy permits the motion of all of the valence electrons of the atoms as if they were free electrons and produces the electronic structure of a degenerated free electron gas among ions arranged at random. To corroborate such an assumption, after the estimation of the Hall coefficient, the magnetic susceptibility of molten pure metals and their alloys was measured and the results were compared with the theoretical values computed on the basis of the free electron gas model.

2. The magnetic susceptibility of moltn metals is exceedingly weak, having a value of the order of $10^{-7}$. to $10^{-8}$, so that the measurement was performed by means of an extremely sensitive balance method using a balance of which the inclination was read after being converted into a capacity charge.
3. The metals used in this experiment were $99.9 \%$ purity or higher, and consisted of $\mathrm{Ag}, \mathrm{Cd}, \mathrm{In}, \mathrm{Sn}, \mathrm{Pb}, \mathrm{Bi}$ and $\mathrm{Sb}$ as pure metals, and of $\mathrm{Bi}-\mathrm{Ag}, \mathrm{Bi}-\mathrm{Cd}, \mathrm{Bi}-\mathrm{In}, \mathrm{Bi}-$ $\mathrm{Sn}, \mathrm{Sn}-\mathrm{Pb}$ and $\mathrm{Sb}-\mathrm{Pb}$ as alloys.

4. Under the above assumption, the total magnetic susceptibility $\chi$ of the molten metals is given by the sum of the shares contributed by electrons $\left(\chi_{\text {electron }}\right)$ and by ion cores $\left(\chi_{\text {ion }}\right)$, the former being calculated by Landau's foemula for free electron gas and for the latter, the values for tested metals were obtained from Mott's and Van Vleck's tables. The sum of these values were used as theoretical values for comparision with the measured values.

The results showed that the measured values of magnetic susceptibility were always independent of the temperature in the molten state, as required by theoretical deduction, and agreed well with the calculated values within the experimental error.

These results prove the reliability of the assumption that the electronic structure of metals in the molten state can be approximated by the free electron gas model. 